\title{
A DECOMPOSITION OF THE RAVIART-THOMAS FINITE ELEMENT INTO A SCALAR AND AN ORIENTATION-PRESERVING PART
}

\author{
Fleurianne BERTRAND ${ }^{12}$ \\ ${ }^{2}$ University of Twente \\ Drienerlolaan 5, 7522 NB Enschede, Netherlands \\ f.bertrand@utwente.nl
}

Key words: mixed finite element methods, Raviart-Thomas element

\begin{abstract}
This contribution considers the conforming finite element discretizations the vector-valued function space $H(d i v, \Omega)$ in 2 and 3 dimensions. A new set of basis functions on simplices is introduced, using a decomposition into an orientation setting part with the edgewise constant normal flux as a degree of freedom and an orientation preserving higher-order part. As a simple combination of lowest-order Raviart-Thomas elements and higher order Lagrange-elements, the basis is suited for fast assembling strategies.
\end{abstract}

\section{Introduction}

Accurate flux and stress approximations are of crucial interest in many applications, although the standard Galerkin approximation usually minimize an energy depending on a primal variable. The divergence of those approximation does usually not belong to the Sobolev space $H(\operatorname{div}, \Omega)$, consisting of vector fields for which the components and the weak divergence are square-integrable. A lot of attention has therefore been devoted to the reconstruction of the flux from a primal formulation. The reconstruction procedures for fluxes are also of particular importance for a posteriori error estimation and have a long history with ideas dating back at least as far as [17] and [18]. A unified framework for Stokes is presented in [16], polynomial-degree robustness is shown in [13] and extensions to three space dimensions in [14].

An alternative approach uses flux-based variational formulations involving the flux as an independent variable approximated in a suitable $H$ (div)-conforming finite element spaces. Such approaches may either lead to a saddle-point problem or a symmetric positive definite system. The first one, have been intensively studied and we refer to [8] for an overview. The second type includes in particular the LeastSquares Method (see [7] for a comprehensive overview) and the discontinuous Petrov-Galerkin method, introduced in a series of papers $[9,10,11]$.

The use of $H(d i v, \Omega)$-conforming finite elements is therefore crucial in challenging applications. Throughout this paper, a regular triangulation $\mathcal{T}$ of a domain $\Omega \subset \mathbf{R}^{d}$ is considered, and $P_{k}(\mathcal{T})$ denotes the space of discontinuous polynomials of degree $k$ with respect to $\mathcal{T}$. Recall that for a finite element family to be $H(d i v, \Omega)$-conforming, the normal component must be continuous. For a given polynomial degree $k \geq 0$

\footnotetext{
${ }^{1}$ Acknowledgments: The author gratefully acknowledge support by the Deutsche Forschungsgemeinschaft in the Priority Program SPP 1748 Reliable simulation techniques in solid mechanics, Development of non standard discretization methods, mechanical and mathematical analysis under the project number BE 6511/1-1.
} 
the smallest polynomial space from which the divergence maps onto $P_{0}(\mathcal{T})$ is the so-called the so-called Raviart-Thomas element, denoted by $R T_{k}(\mathcal{T})$ and introduced in [?].

The construction of the basis function and the choice of the degrees of freedom for the Raviart-Thomas element are an important issue in view of fast assembling and computations. They are less evident than for the standard element, as not all degrees of freedom are pointvalues. Computational bases for $H(\operatorname{div}, \Omega)$ with Lagrangian property are derived in [15], althought the autor restrict himself to the two-dimensional case. This basis is defined on the reference triangle and the continuity of the normal component of the approximation across the edges in the triangulation is satisfied by the use of the Piola transformation and the Lagrangian property of the basis functions. However, using an affine transformation to map a reference triangle on any triangle in the triangulation is less appropriate regarding an adaptive mesh refinement such that an alternative approach forming local bases on any triangle in the triangulation is proposed in [2]. A construction of basis functions which implies sparse element matrices has been developped in [6], where the authors are concerned with fast assembling and fast matrix-vector multiplications.

The efficient assembly of $H(d i v)$ is an important issue, discussed e.g. in [19], where the authors propose an implementing strategy relying on a decomposition of the element tensor into a precomputable reference tensor and a mesh-dependent geometry tensor. An alternative effective and fexible way to assemble finite element stiffness and mass matrices with edge elements is proposed in [1].

The aim of this paper is to propose a decomposition of the Raviart-Thomas space into a scalar and an orientation-preserving part, as this will increase the speed of the assembling strategies. In fact, the orientation of the basis function can be defined once and for all for the lowest-order Raviart-Thomas space, and be conserved in the higher-order aase. Such a decomposit
the effect of approximated flux boundary conditions (see [5]). For th
Raviart-Thomas elements (see [4]) this decomposition can be used
the parametric mapping, since it is then sufficient to apply the H(div
order orientation-preserving part and to use the standard isoparame
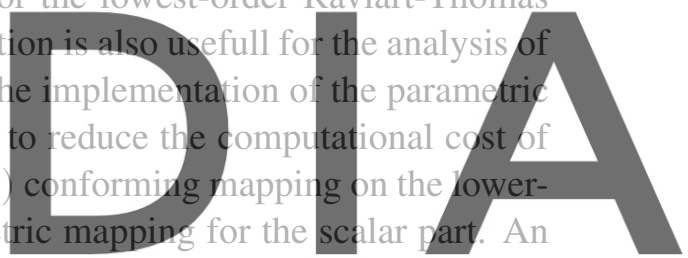

other application of this decomposition is the derivation of hierarchical error estimator, simplifying the

Register for fysis and humbing/computatipnal gist down to download the version without the watermark The main result of this paper is that given a basis of $R T_{0}(\mathcal{T})$, it is possible to find a set of basis functions for $R T_{k}(T)$, that are given as a product of the $R T_{0}(\mathcal{T})$ basis functions and a scalar function. This means that the lowest order basis functions can be considered as the orientation part of all the cell-based higher-order basis functions. Since these are easily computable and since the scalar part can be seen as a Lagrange basis function this implies a reduction of the computational cost. This paper is organized as follows: section 2 recalls some properties of the Raviart-Thomas element while in section 3 the orientation-preserving basis function are provided. In section 4, a basis retaining the orientation is constructed for the quadratic case. Finally, the results are extended to the higher-order case in section 5.

\section{The Raviart-Thomas element}

In this section, we review the different definitions of the degrees of freedom. Note that even the indexing of the Raviart-thomas space is not consistent in the literature. We choose to work with the following definition of the Raviart-Thomas element:

$$
R T_{k}(T)=\left(\mathcal{P}_{k}(T)\right)^{d}+\mathbf{x} \mathcal{P}_{k}(T)
$$




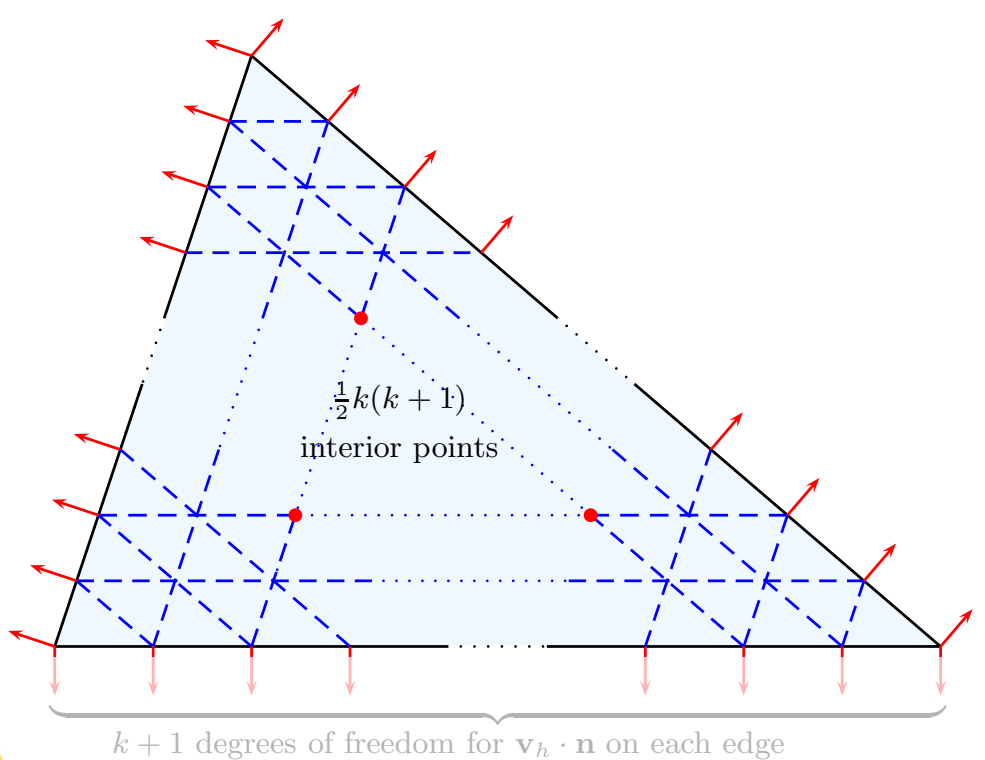

Figure 1: Degrees of freedom for the Raviart-Thomas element

forming the Raviart-Thomas space

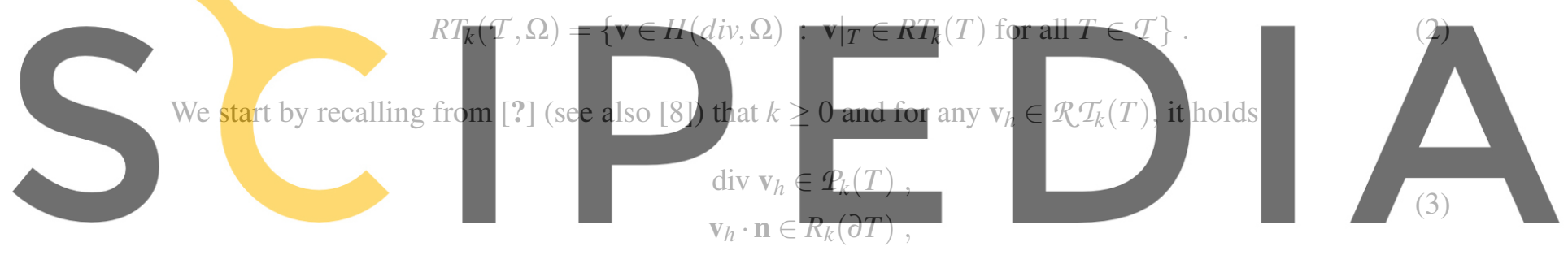

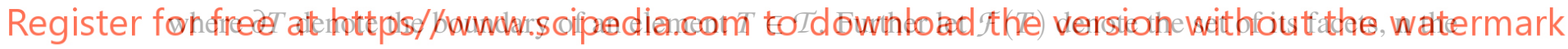
outward oriented normal and

$$
R_{k}(\partial T)=\left\{\phi \in L^{2}(\partial T):\left.\phi\right|_{e} \in \mathcal{P}_{k}(e) \quad \forall e \in \mathcal{F}(T)\right\}
$$

the polynomial space on the facets. Recall that if

$$
\begin{aligned}
& \int_{\partial T}\left(\mathbf{n} \cdot \mathbf{v}_{h}\right) p_{k} d s, \quad \forall p_{k} \in R_{k}(\partial T) \\
& \int_{T} \mathbf{v}_{h} \cdot \mathbf{p}_{k-1} d \mathbf{x}, \quad \forall \mathbf{p}_{k-1} \in\left(P_{k-1}(T)\right)^{d}
\end{aligned}
$$

holds for $\mathbf{v}_{h} \in R T_{k}(T)$, then $\mathbf{v}_{h}=\mathbf{0}$. As represented in figure 2 and 3 for $k=0$ and $k=1$ (see [12] as well), this motivate the fact that the degrees of freedom $\boldsymbol{\Sigma}_{k}\left(\mathbf{v}_{h}\right)=\boldsymbol{\Sigma}_{\mathbf{R}, k}^{\mathbf{n}}\left(\mathbf{v}_{h}\right) \cup \boldsymbol{\Sigma}_{\mathbf{P}, k}^{\text {int }}\left(\mathbf{v}_{h}\right)$ are typically given 
by

$$
\begin{aligned}
& \Sigma_{\mathbf{R}, k}^{\mathbf{n}}\left(\mathbf{v}_{h}\right)=\left\{\int_{\partial T}\left(\mathbf{n} \cdot \mathbf{v}_{h}\right) p_{k} d s: p_{k} \in \mathbf{R}\right\} \\
& \Sigma_{\mathbf{P}, k}^{\text {int }}\left(\mathbf{v}_{h}\right)=\left\{\int_{T} \mathbf{v}_{h} \cdot \mathbf{p}_{k-1} d \mathbf{x}: \mathbf{p}_{k-1} \in \mathbf{P}\right\}
\end{aligned}
$$

where $\mathbf{R}$ and $\mathbf{P}$ are basis for $R_{k}(\partial T)$ and $\left(P_{k-1}(T)\right)^{d}$ respectively. Note that the degrees of freedom depend on this basis. Denoting the standard conforming Lagrangian basis function on a element $E$ with $\mathcal{L}^{k}(E)$, one possible choice are

$$
\mathbf{R}=\left\{q \in L^{2}(\partial T):\left.q\right|_{e} \in \mathcal{L}^{k}(e) \quad \forall e \in \mathcal{F}(T)\right\}
$$

and $\mathbf{P}=\mathcal{L}^{k-1}(\boldsymbol{T})$. Constructing the set of functions

$$
\left\{\mathbf{v}_{i} \in R T_{k}(T):\left\{\Sigma_{k}\left(\mathbf{v}_{i}\right)\right\}_{j}=\delta_{i j}\right\}_{i=1}^{\operatorname{dim} R T_{k}(T)}
$$

leads to a dual basis of $R T_{k}(T)$. Throughout this paper, $R T_{k}^{\mathrm{n}}(\mathcal{T})$ denotes the linear combination of the basis functions assiociated with (6a) such that

$$
R T_{k}(\mathcal{T}, \Omega)=R T_{k}^{\mathrm{n}}(\mathcal{T}) \bigoplus R T_{k}^{\mathrm{int}}(\mathcal{T})
$$

holds. Note that for $k=0, R T_{k}^{\text {in }}$ $\operatorname{dim} R T_{k}($
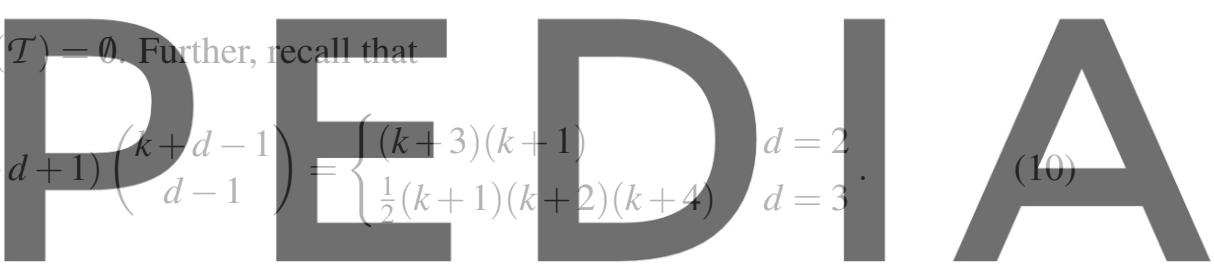

Due to $\mathbb{P}^{k-1}(K) \subset R T_{k}(T)$ and $\mathbf{v}_{h} \cdot \mathbf{n} \in R_{k}(\partial T)$ for $\mathbf{v}_{h} \in R T_{k}(T)$, an alternative way to define the degrees

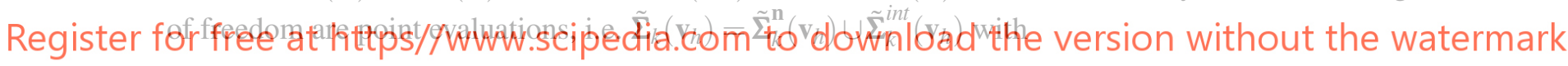

$$
\begin{aligned}
& \tilde{\Sigma}_{k, \Xi}^{\mathrm{n}}\left(\mathbf{v}_{h}\right)=\left\{\left.\left(\mathbf{v}_{h}\left(\xi_{i}\right) \cdot \mathbf{n}\right)\right|_{e}: \xi_{i} \in \Xi, e \in \mathcal{F}(T)\right\} \\
& \tilde{\boldsymbol{\Sigma}}_{k, \mathbf{H}}^{\text {int }}\left(\mathbf{v}_{h}\right)=\left\{\mathbf{v}_{h, j}\left(\boldsymbol{\eta}_{i}\right): \boldsymbol{\eta}_{i} \in \mathbf{H}\right\}_{j=1}^{d}
\end{aligned}
$$

where $\mathbf{H}$ is a point set on an element $T$ with

$$
\operatorname{dim}(\mathbf{H})=\left(\begin{array}{c}
k-1+d \\
d
\end{array}\right)
$$

and $\Xi$ is a point set on a facet, with

$$
\operatorname{dim}(\mathbf{\Xi})=\left(\begin{array}{c}
k-1+d \\
k
\end{array}\right)
$$

Now, constructing the set of functions

$$
\left\{\mathbf{v}_{i} \in R T_{k}(T):\left\{\tilde{\boldsymbol{\Sigma}}_{k}\left(\mathbf{v}_{i}\right)\right\}_{j}=\delta_{i j}\right\}_{i=1}^{\operatorname{dim} R T_{k}(T)}
$$




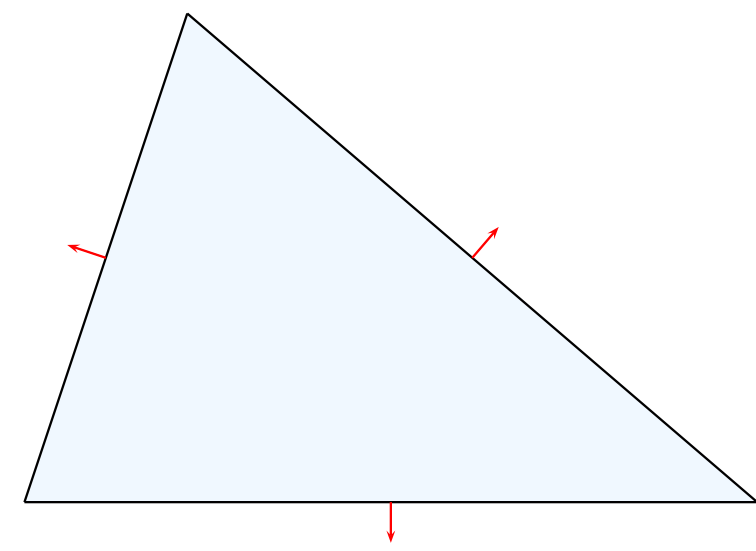

(a) $k=0$

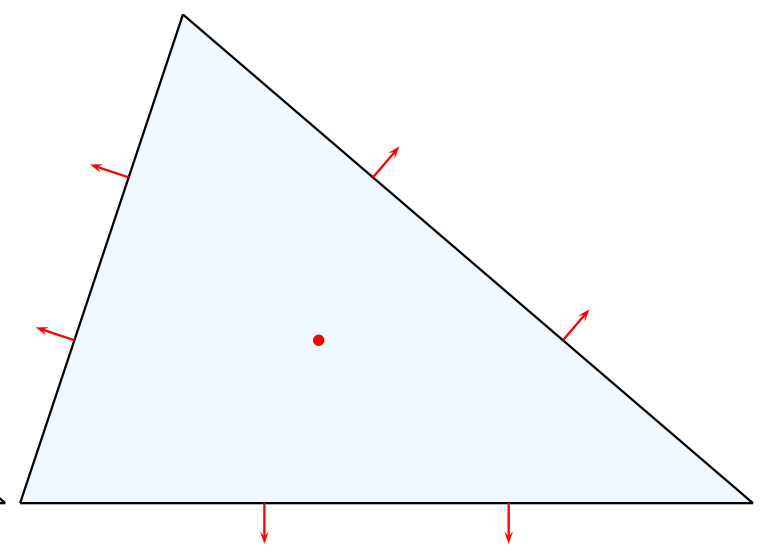

(b) $k=1$

Figure 2: Degrees of freedom for the Raviart-Thomas element for $k=0$ and $k=1$

leads to basis of $R T_{k}(T)$. For $k \geq 1$, the points for $(6 \mathrm{~b})$ can be chosen as the interior points of the Lagrange element of type $k+d$, each point represents $d$ degrees of freedom (see figure 1). Note that in fact, there is no need to enforce the evaluations of the different components at the same point, such that $\overline{\boldsymbol{\Sigma}}_{k}\left(\mathbf{v}_{h}\right)=\tilde{\Sigma}_{k}^{\mathbf{n}}\left(\mathbf{v}_{h}\right) \cup \bar{\Sigma}_{k}^{i n t}\left(\mathbf{v}_{h}\right)$ with

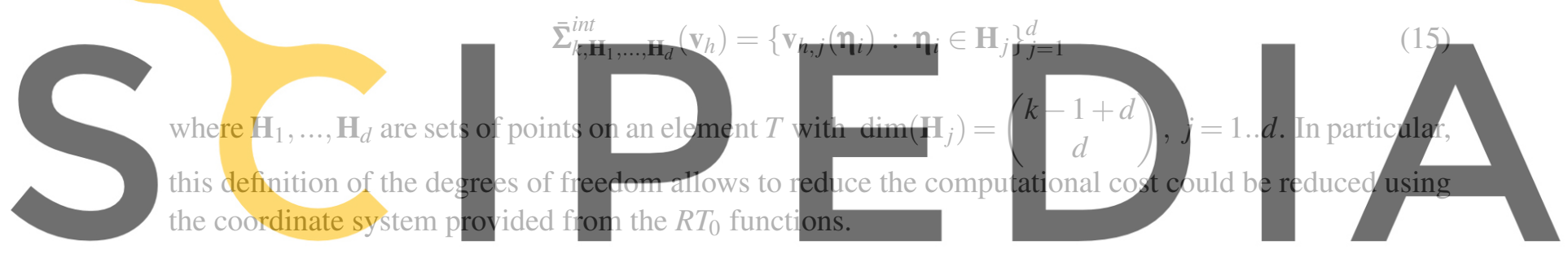

Register for $^{3}$ Orientation preserving Basis Functions

for free at https//www.scipedia.com to download the version without the watermark

[3] provides three short Matlab implementations of the lowest-order Raviart-Thomas mixed finite ele-

ments for the numerical solution of a Laplace equation with mixed Dirichlet and Neumann boundary

conditions. In particular, a section is devoted to the edge-basis functions for the lowest-order Raviart-

Thomas finite elements. Although these results are written down for the $2 \mathrm{D}$ case, the representation of a Raviart-Thomas function directly carries over to three dimensions. In the lowest-order case, the RaviartThomas Function space $R T_{0}(\mathcal{T})$ is given by facet-functions, i.e. there is a basis $\hat{\Psi}=\left\{\hat{\phi}_{F}\right\}_{F \in \mathcal{F}}$, where $\phi_{F}$ lives on the two adjacent elements of $F$. This together with the $H($ div $)$ conformity implies that the facets will be oriented. Therefore, for a given facet $F \in \mathcal{F}$ a left and a right triangle denoted by $T_{+}$and $T_{-}$are defined, corresponding to left and right $d$-th points denoted by $P_{+}$and $P_{-}$. This defines one for all the orientation of the given edge, and the purpose of the next section is to retain the orientation in the corresponding basis function. The basis functions are given by

$$
\hat{\psi}_{F}(\mathbf{x})=\left\{\begin{array}{lc}
\frac{|F|}{2\left|T_{+}\right|}\left(\mathbf{x}-P_{+}\right) & \text {on }\left|T_{+}\right| \\
-\frac{|F|}{2\left|T_{-}\right|}\left(\mathbf{x}-P_{-}\right) & \text {on }\left|T_{-}\right| \\
0 & \text { elsewhere }
\end{array}\right.
$$




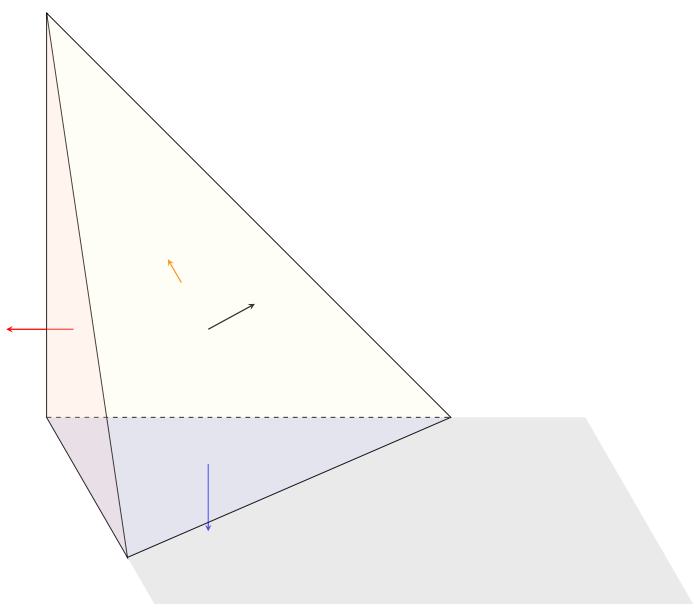

(a) $k=0$

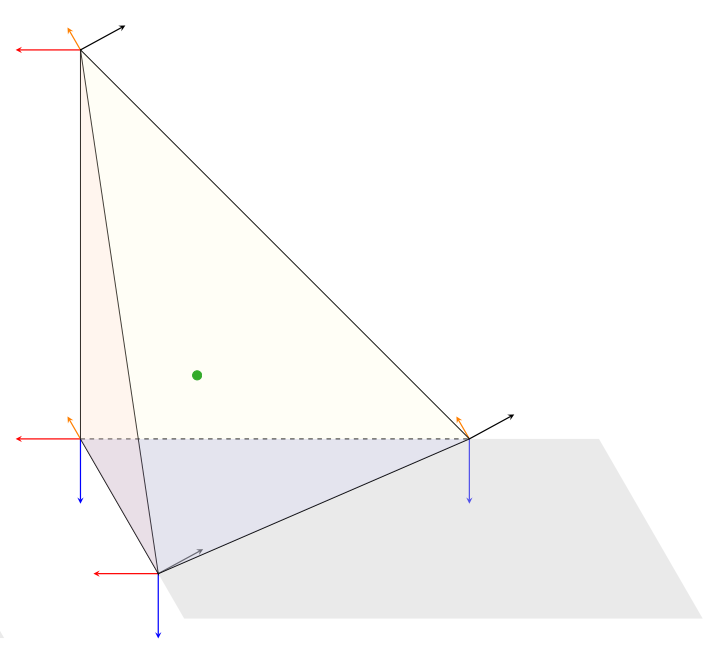

(b) $k=1$

Figure 3: Degrees of freedom for the Raviart-Thomas element $(d=3)$

where $|F|$ denotes the measure of the facet $F$ and $|T|$ denotes the measure of the element $T$. These basis functions correspond to the nodal set of degrees of freedom $\tilde{\Sigma}_{0, \cdot}^{\mathrm{n}}=\Sigma_{\tilde{\mathbf{R}}^{0}, k}^{\mathrm{n}}$, with

$$
\tilde{\mathbb{R}}^{0}=\left\{q: e \mapsto \mathbb{R}: q(\mathbf{x})=|F|^{-1}\right\}_{e \in \mathcal{F}(T)} .
$$

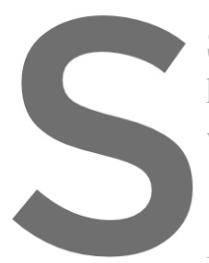

Since the normal compon

have to be specified. In th

with the points of an edge $E$ by $\lambda$
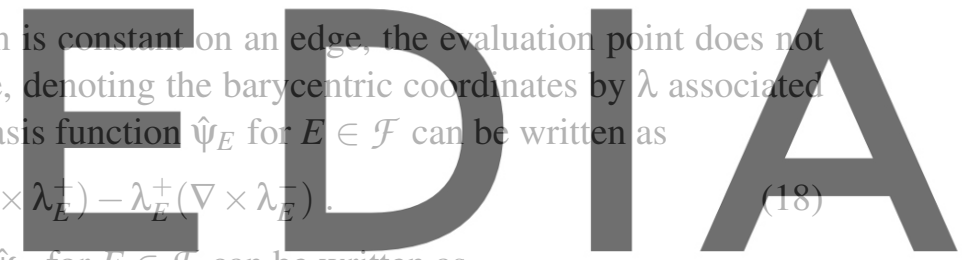

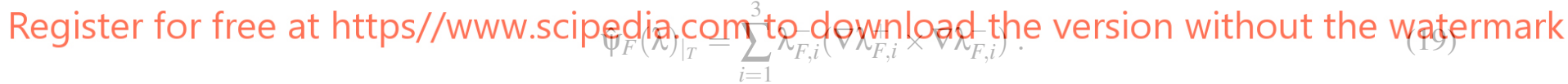

where $\left\{\lambda_{F, i}\right\}_{i=1}^{3}$ denotes the barycentric coordinates associated with the $i-t h$ vertice of the face $F$, and $\nabla \lambda_{F, i}^{+}$and $\nabla \lambda_{F, i}^{-}$its adjacent points on the face.

The alternative definition of the degrees of freedom with the moments leads to

$$
\hat{\varphi}_{F}(\mathbf{x})=\left\{\begin{array}{lc}
\frac{1}{2 \mid T_{+}}\left(\mathbf{x}-P_{+}\right) & \text {on }\left|T_{+}\right| \\
-\frac{1}{2\left|T_{-}\right|}\left(\mathbf{x}-P_{-}\right) & \text {on }\left|T_{-}\right| \\
0 & \text { elsewhere }
\end{array}\right.
$$

corresponding to $\Sigma_{\mathbf{R}^{0}, k}^{\mathbf{n}}$, with

$$
\mathbf{R}^{0}=\{q: e \mapsto \mathbf{R}: q(\mathbf{x})=1\}_{e \in \mathcal{F}(T)} .
$$

Throughout the further sections, the choice of the set of basis functions for $R T_{0}$ does not matter, the decomposition into a orientation-part and the scalar part will conserve the scaling of the $R T_{0}$ basis functions into the higher-order case either way. 


\section{Quadratic Raviart-Thomas Element}

In this section, a basis for the facet-based shape functions $R T_{2}^{\mathbf{n}}(T)$ is proposed. Recall that we want to conserve the orientation defined by the lowest-order facet-based functions. Therefore, define the linear scalar-valued polynomials $p_{F, P, T}: T \mapsto \mathbf{R}$ such that

$$
\begin{aligned}
p_{F, P, T}(P) & =1 \\
p_{F, P, T}\left(P_{m}(T)\right) & =0 \text { and } \\
p_{F, P, T}\left(P_{i}\right) & =0 \text { for } P_{i} \in Q(F) \backslash P .
\end{aligned}
$$

where $P_{m}(T)$ denote the center of gravity of the element $T$ and $Q(F)$ the set of the vertices of the facet $F$. Note that these functions are unique and correspond to a Lagrange Basis on a uniform refined mesh. This leads to the following lemma.

Lemma 1. A basis for $R T_{2}^{\mathbf{n}}(T)$ is given by $\left\{\boldsymbol{\psi}_{P_{i}, F}\right\}_{F \in \mathcal{T}, i=1, \ldots, d}$, with

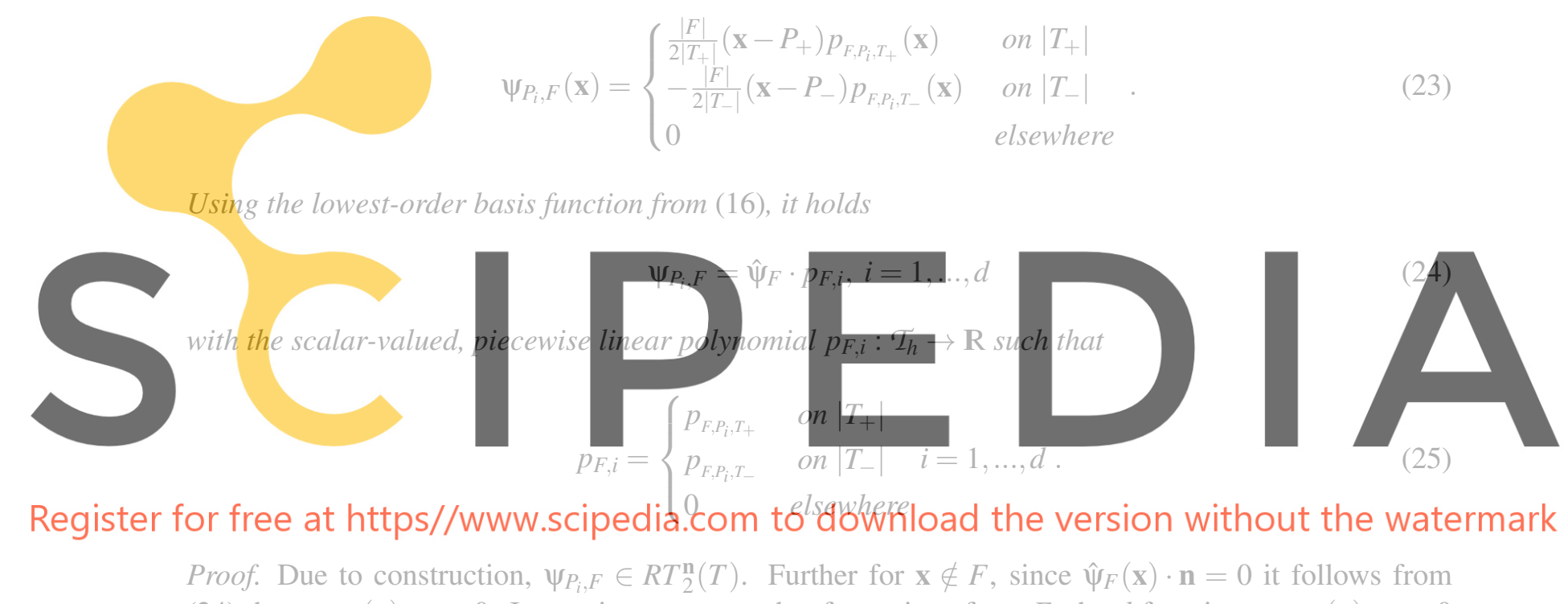

(24) that $\psi_{P_{i}, F}(\mathbf{x}) \cdot \mathbf{n}=0$. It remains to proove that for a given facet $F$, the $d$ functions $\psi_{P_{i}, F}(\mathbf{x}) \cdot \mathbf{n}=0$ for $P_{i} \in Q(F)$ are linearly independent. Due to (24), this is equivalent to the fact that the $d$ functions $p_{F, i}, i=1, \ldots, d$ are linearly independent for a given facet $F$, and this is ensure by the conditions (22), and the fact that the triangulation is regular, since these are $d+1$ conditions for a linear polynomial in $\mathbf{R}^{d}$.

Note that in the two-dimensional case, due to symmetric properties, it holds

$$
\begin{aligned}
p_{F, P, T}(P) & =1 \\
p_{F, P, T}\left(P_{m}(T)\right) & =0 \text { and } \\
p_{F, P, T}\left(P_{+}(F)\right) & =-1 .
\end{aligned}
$$

To obtain an orientation preserving basis, it remains to construct a basis for $R T_{2}^{\text {int }}(\mathcal{T})$, using the lowestorder basis functions. Therefore for a facet $F$ of an element $T \in \mathcal{T}_{h}$ consider the scalar-valued linear 


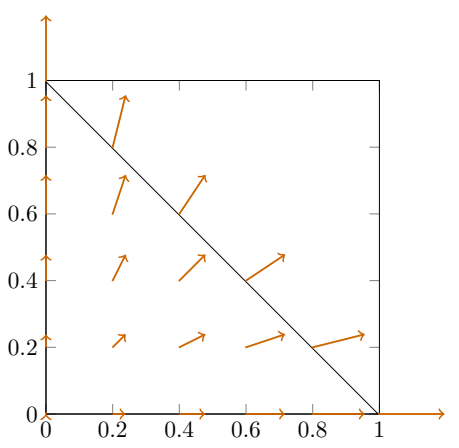

(a) $\boldsymbol{\psi}_{1}=(x, y)^{\top}$

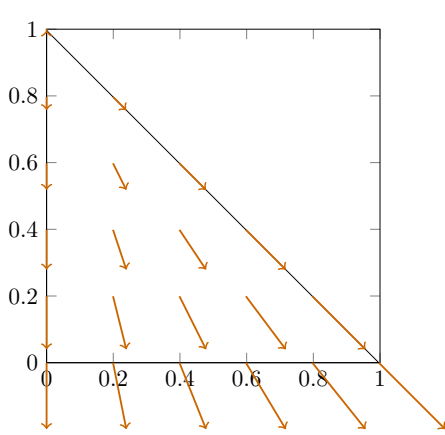

(b) $\boldsymbol{\Psi}_{2}=(x-1, y)^{\top}$

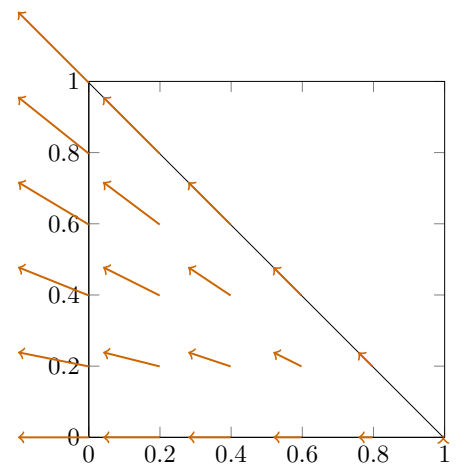

(c) $\boldsymbol{\Psi}_{3}=(x, y-1)^{\top}$
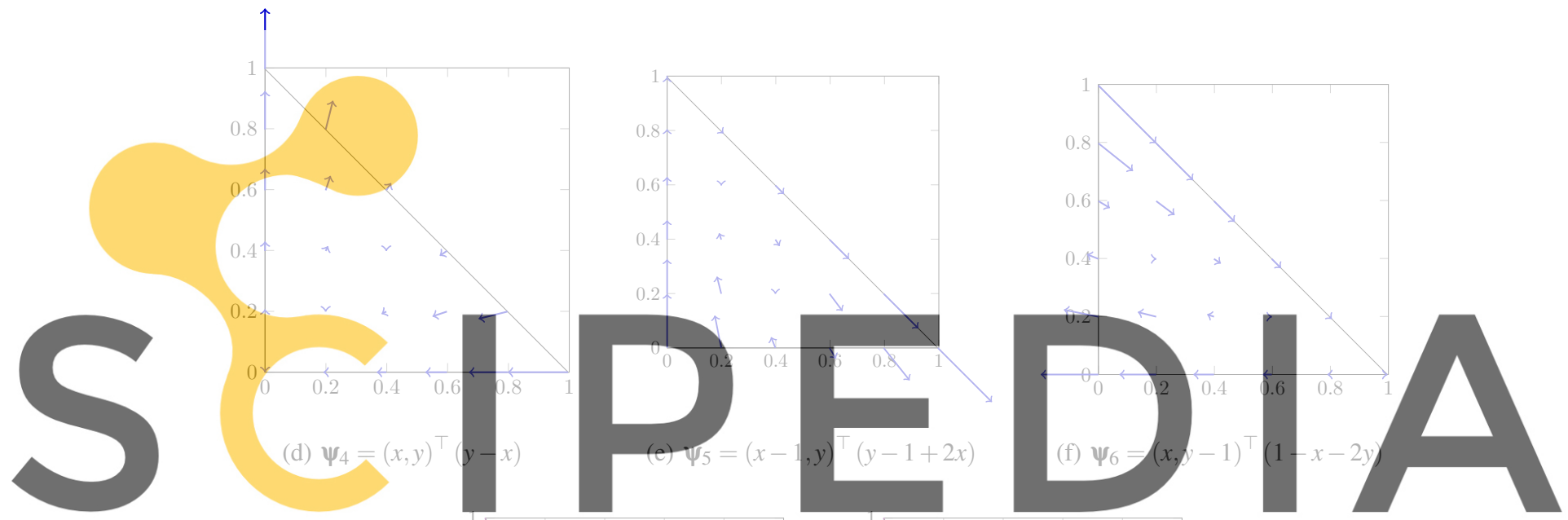

Register for free at https//www.scipedia.com to download the version without the watermark

(g) $\boldsymbol{\Psi}_{7}=(3 x(-2+2 x+y), 3 y(-1+2 x+y))^{\top}$

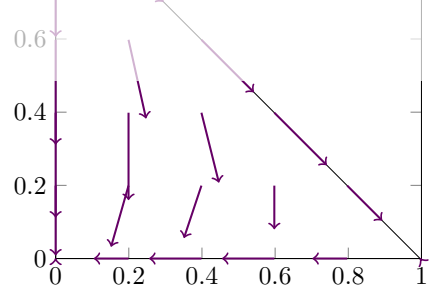

(h) $\boldsymbol{\Psi}_{8}=(3 x(x+2 y-1), 3 y(-2+x+2 y))^{\top}$

Figure 4: Example for ansatzfunctions on reference triangle

polynomial $p_{F, T}: T \rightarrow \mathbf{R}$ such that

$$
\begin{aligned}
p_{F, T}\left(P_{i}(F)\right) & =0, i \in\{1, \ldots, d\} \\
p_{F, T}\left(P_{m}(T)\right) & =1
\end{aligned}
$$

and let $i_{\min }(T)$ be the index such that $P_{i_{\min }(T)}$ is the vertice of $T$ with minimal angle. Note that $p_{F, T}$ 
althought correspond to a Lagrange Basis on a uniform refined mesh, such that the following lemma ensures a simple computation of the basis functions for $\mathcal{T}$.

Lemma 2. A basis for $R T_{2}^{\text {int }}(\mathcal{T})$ is given by $\left\{\psi_{i, T}\right\}_{T \in \mathcal{T}, i \in\{1,2,3,4\} \backslash\left\{i_{\min }(T)\right\}}$ with

$$
\psi_{i, T}=\hat{\psi}_{F\left(P_{i}, T\right)} \cdot p_{F, T}
$$

where $F\left(P_{i}, T\right)$ denotes the facet of $T$ which intersection with $P_{i}$ is empty.

Proof. Since $p_{F\left(P_{i}\right), T}=0$ for each vertices of the facet $F$ and $\hat{\psi}_{F\left(P_{i}, T\right)}$ vanishes for the remaining verctice of the element $T, \psi_{i, T}$ vanishes at all vertices of $T$. This implies that the normal component of $\psi_{i, T}$ vanishes on all facet, such that it remains to check that the $d$ functions $\psi_{i, T}$ are linearly independent for a given $T$, and that holds due to the fact that $\hat{\psi}_{F\left(P_{i}, T\right)}, i \in\{1,2,3,4\} \backslash\left\{i_{\min }(T)\right\}$ are linearly independent.

\section{Higher order case}

The results from the previous section can be extended to higher order elements. Therefore recall that a Lagrangian element of order $k+d$ has

$$
N_{I}(k)=\left(\begin{array}{c}
k-1+d \\
d
\end{array}\right)
$$

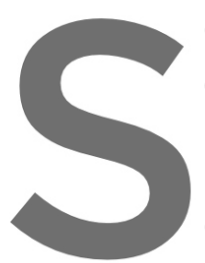

degrees of Freedom located

corresponding to these $\left(\begin{array}{c}d-1+k \\ k\end{array}\right)$ denotes the

define a unique scalar-valued polynomial of order $h$ and $\left\{R_{k}(F)\right\}_{F \in \mathcal{F}(T)}$.
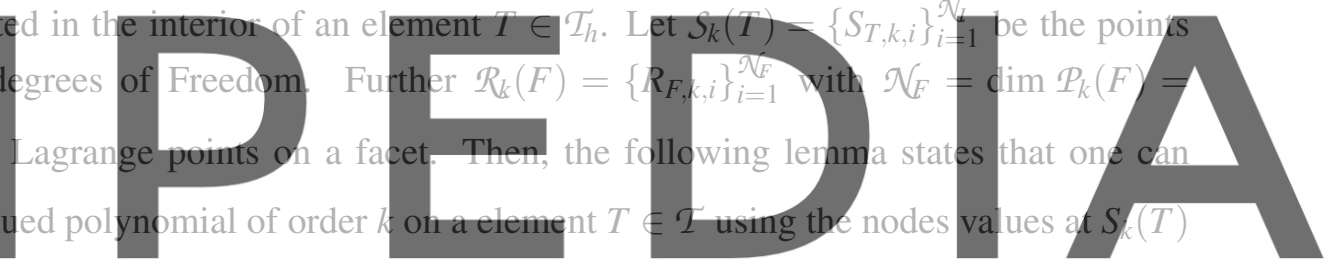

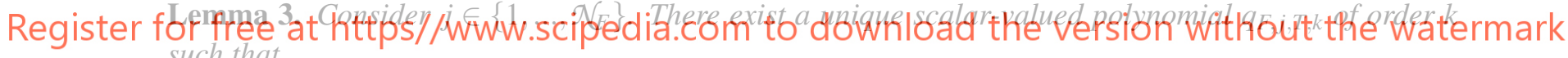
such that

$$
\begin{aligned}
& q_{F, j, T, k}\left(R_{F, k, j}\right)=1 \\
& q_{F, j, T, k}\left(R_{F, k, i}\right)=0 \text { for } i=1, \ldots, \mathcal{N}_{F}, i \neq j \text { and } \\
& q_{F, j, T, k}\left(S_{T, k, i}\right)=0 \text { for } i=1, \ldots, \mathcal{N}_{I} .
\end{aligned}
$$

Proof. Since all points are distinct, the equations (30) corresponds to $\mathcal{N}_{F}+\mathcal{N}_{I}$ equations and it is sufficient to prove that $\operatorname{dim} \mathcal{P}_{k}(T)=\mathcal{N}_{F}+\mathcal{N}_{I}$. In fact, it holds

$$
\begin{aligned}
\mathcal{N}_{F}+\mathcal{N}_{I}-\operatorname{dim} \mathcal{P}_{k}(T) & =\left(\begin{array}{c}
d-1+k \\
k
\end{array}\right)+\left(\begin{array}{c}
k-1+d \\
d
\end{array}\right)-\left(\begin{array}{c}
d+k \\
k
\end{array}\right) \\
& =\frac{(d-1+k) !}{(d-1) !(k-1) !}\left(\frac{1}{k}+\frac{1}{d}-\frac{d+k}{k d}\right) \\
& =0 .
\end{aligned}
$$


Note that these functions again correspond to a Lagrange Basis on a uniform refined mesh, such that the following theorem implies a possible fast implementation of the facet-based Raviart-Thomas basis functions.

Theorem 1. A basis for $R T_{k}^{\mathbf{n}}(T)$ is given by $\left\{\psi_{i, F, k}\right\}_{F \in \mathcal{F}, i=1 . . d}$, with

$$
\psi_{i, F, k}(\mathbf{x})= \begin{cases}\frac{|F|}{2\left|T_{+}\right|}\left(\mathbf{x}-P_{+}\right) q_{F, i, T_{+}, k}(\mathbf{x}) & \text { on }\left|T_{+}\right| \\ -\frac{|F|}{2\left|T_{-}\right|}\left(\mathbf{x}-P_{-}\right) q_{F, i, T_{-}, k}(\mathbf{x}) & \text { on }\left|T_{-}\right| \quad i=1, \ldots, d . \\ 0 & \text { elsewhere }\end{cases}
$$

Using the lowest-order basis function, it holds

$$
\psi_{i, F, k}=\hat{\psi}_{F} \cdot q_{F, i, k}, i=1, \ldots, d
$$

with

$$
q_{F, i, k}= \begin{cases}q_{F, i, T_{+}, k} & \text { on }\left|T_{+}\right| \\ q_{F, i, T_{-}, k} & \text { on }\left|T_{-}\right| \quad i=1, \ldots, d . \\ 0 & \text { elsewhere }\end{cases}
$$

Proof. Similarly to the quadratic case, the construction of the basis function implies $\psi_{i, F, k} \in R T_{k}^{\mathrm{n}}(T)$.

Further for $\mathbf{x} \notin F$, since $\hat{\psi}_{\mathbf{i}, F, k}(\mathbf{x}) \cdot \mathbf{n}=0$ it follows from $(32)$ that $\psi_{i, F}(\mathbf{x}) \cdot \mathbf{n}=0$. It remains to prove that

for a given facet $F$, the $d$

is equivalent to the fact th

and this is the statement of lemm

It remains to construct a basis for $R T_{k}^{\text {int }}(T)$. Therefore, for a p

polynomial $q_{F, T, S}: T \rightarrow \mathbb{R}$ of order $k$ such that

Register for free at https//www.scipedia.com to download the version without the watermark

$$
\begin{aligned}
& q_{F, T, S, k}(P)=0, P \in \mathbb{R}_{k}(F) \\
& q_{F, T, S, k}(P)=0, P \in \mathcal{S}_{k}(T) \backslash S .
\end{aligned}
$$

These polynomial functions leads to a basis of $R T_{k}^{\text {int }}(T)$, as the following theorem states.

Theorem 2. $\left\{\psi_{i, j, T}\right\}_{T \in \mathcal{T}, i \in\{1,2,3,4\} \backslash\left\{i_{\text {min }}\right\}, j=1, \ldots, \mathcal{N}_{I}}$ with

$$
\psi_{i, j, T}=\left.\hat{\psi}_{F\left(P_{i}\right)}\right|_{T} \cdot q_{F\left(P_{i}\right), T, S_{T, k, i}, k}
$$

define a basis for $R T_{k}^{\text {int }}(T)$.

Proof. Recall that the normal component of $\hat{\psi}_{F\left(P_{i}\right.}$ vanishes on all facet of $T$ but F. Thus, $q_{F, T, S, k}=0$ on $F$ implies that the normal component of $\psi_{i, j, T}$ vanishes on all facet, such that it remains to check that the $d \cdot \mathcal{N}_{i}$ functions $\psi_{i, j, T}$ are linearly independent for a given $T$. This is ensured by the combiation of the linearly independency of $\hat{\psi}_{F\left(P_{i}, T\right)}, i \in\{1,2,3,4\} \backslash\left\{i_{\text {min }}(T)\right\}$ and the $N_{i}$ nodes values. 


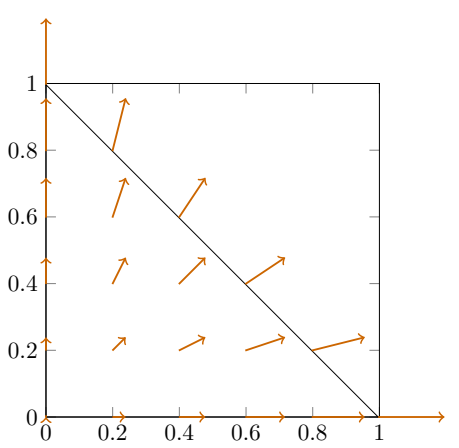

(a) $\boldsymbol{\psi}_{1}=(x, y)^{\top}$

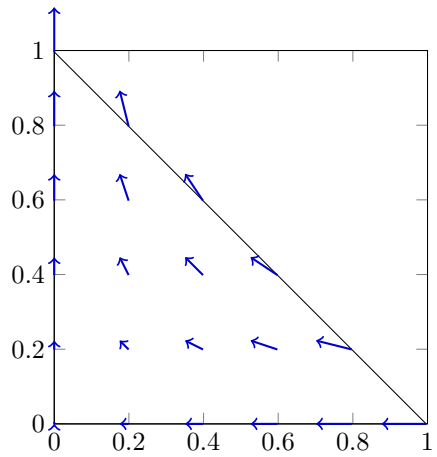

(d) $\boldsymbol{\Psi}_{4}=(-3 x, 3 y)^{\top}$

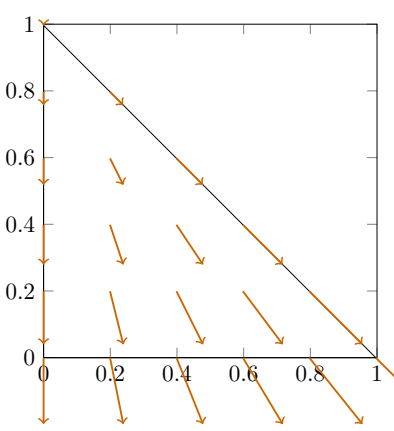

(b) $\boldsymbol{\Psi}_{2}=(x-1, y)^{\top}$

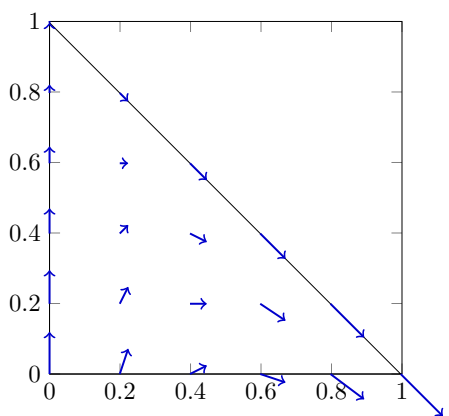

(e) $\left.\boldsymbol{\Psi}_{5}=(3 x,-6 x-3 y+3)\right)^{\top}$

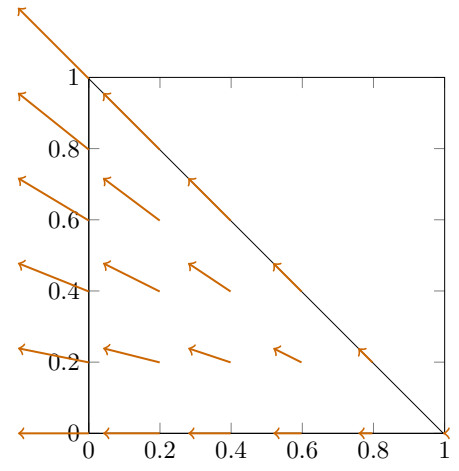

(c) $\boldsymbol{\Psi}_{3}=(x, y-1)^{\top}$

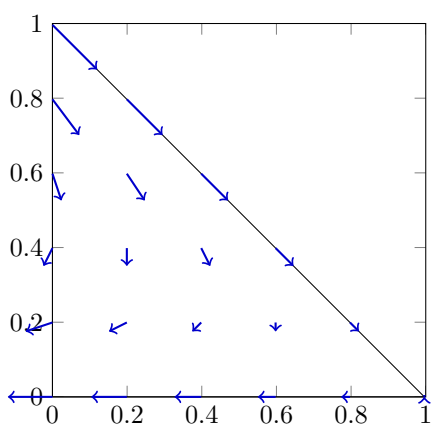

(f) $\left.\boldsymbol{\Psi}_{6}=(3 x+6 y-3,-3 y)\right)^{\top}$

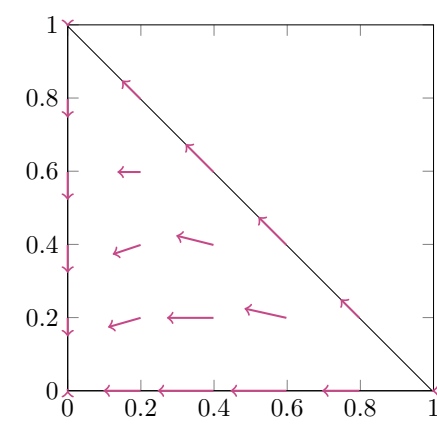

(g) $\boldsymbol{\Psi}_{7}=\left(16 x^{2}+8 x y-16 x, 16 x y+8 y^{2}-8 y\right)$

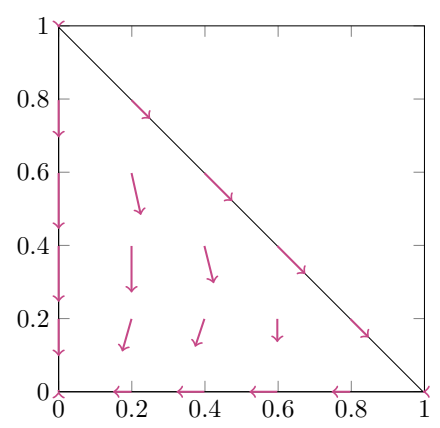

(h) $\boldsymbol{\Psi}_{8}=\left(8 x^{2}+16 x y-8 x, 8 x y+16 y^{2}-16 y\right)^{\top}$

Figure 5: An other set of Ansatzfunctions on reference triangle

\section{REFERENCES}

[1] I. Anjam and J. Valdman. Fast matlab assembly of fem matrices in 2d and 3d: Edge elements. Applied Mathematics and Computation, 267:252-263, 2015.

[2] D. N. Arnold, R. S. Falk, and R. Winther. Finite element exterior calculus, homological techniques, 
and applications. Acta numerica, 15:1-155, 2006.

[3] C. Bahriawati and C. Carstensen. Three matlab implementations of the lowest-order raviart-thomas mfem with a posteriori error control. Computational Methods in Applied Mathematics, 5(4):333$361,2005$.

[4] F. Bertrand, S. Münzenmaier, and G. Starke. First-order system least squares on curved boundaries: Higher-order raviart-thomas elements. SIAM J. Num. Anal, 52(6):3165-3180.

[5] F. Bertrand, S. Münzenmaier, and G. Starke. First-order system least squares on curved boundaries: Lowest-order raviart-thomas elements. SIAM J. Num. Anal, 52(2):880-894, 2014.

[6] S. Beuchler, V. Pillwein, and S. Zaglmayr. Sparsity optimized high order finite element functions for h(curl) on tetrahedra. Advances in Applied Mathematics, 50:749-769, 2013.

[7] P. Bochev and M. Gunzburger. A locally conservative least-squares method for darcy flows. Commun. Numer. Meth. Engrg, 24:97-110, 2008.

[8] D. Boffi, F. Brezzi, and M. Fortin. Mixed Finite Element Methods and Applications. Springer, Heidelberg, 2013.

[9] L. Demkowicz and J. Gopalakrishnan. A class of discontinuous Petrov-Galerkin methods. Part I: the transport equation. Comput. Methods Appl. Mech. Engrg., 199:1558-1572, 2010.

[10] L. Demkowicz and J. Gopalakrishnan. A class of discontinuous Petrov-Galerkin methods. II. Optimal test functions. Numer. Methods Partial Differential Equations, 27:70-105, 2011.

[11] L. Demkowicz, J. Gopalakrishnan, and A. H. Niemi. A class of discontinuous Petrov-Galerkin methods. Part III: Adaptivity. Appl. Numer. Math, 62:496-427, 2012.

[12] A. Ern and J 1 Guermond. Theory and Practice of Finite Elements. Springer, New York, 2004.

[13] A. Ern and M. Vohralík. Polynomial-degree-robust a posteriori error estimates in a unified setting for conforming, nonconforming, discontinuous Galerkin, and mixed discretizations. SIAM J. Numer. Anal, 53:1058-1081, 2015.

[14] A. Ern and M. Vohralik. Stable broken h1 and h(div) polynomial extensions for polynomial-degreerobust potential and flux reconstruction in three space dimensions. Math. Comp, 89:551-594, 2020.

[15] V. J. Ervin. Computational bases for $r t_{k}$ and $b d m_{k}$ on triangles. Computers \& Mathematics with Applications, 64(8):2765-2774, 2012.

[16] A. Hannukainen, R. Stenberg, and M. Vohralík. A unified framework for a posteriori error estimation for the Stokes equation. Numer. Math, 122:725-769.

[17] P. Ladevèze and D. Leguillon. Error estimate procedure in the finite element method and applications. SIAM J. Numer. Anal, 20:485-509, 1983.

[18] W. Prager and J. L. Synge. Approximations in elasticity based on the concept of function space. Quart. Appl. Math, 5:241-269, 1947.

[19] M. E. Rognes, R. C. Kirby, and A. Logg. Efficient assembly of h(div) and h(curl) conforming finite elements. SIAM Journal on Scientific Computing, 31(6):4130-4151, 2009. 